\title{
ULTERIORE STUDIO SULLE SESSE DEL LAGO DI BOLSENA
}

\author{
D. Di FiLippo
}

Richiami teorici. - Chrystal, per lo sviluppo della sua teoria sulle oscillazioni libere, considera il lago come un canale finito di sezione variabile con la profondità, ma non in maniera brusca. Egli pone l'asse $x$ sulla superficie del lago, lungo la linea di massima profondità e l'origine ad una estremità del lago stesso, l'asse $y$ orizzontale e l'asse $z$ verticale, ed inoltre indica con $S(x)$ l'area di una generica sezione normale all'asse $x$, e con $b(x)$ la corrispondente ampiezza alla superficie libera. Considera infine trascurabili, dopo un tempo $t$, gli spostamenti di una particella, soggetta ad una certa azione, lungo l'asse $y$ e chiama con $\xi$ e $\zeta$ rispettivamente gli spostamenti orizzontali secondo l'asse $x$ e quelli verticali secondo $z$.

Scritta l'equazione della continuità e tenuto conto dell'equazione fendamentale dell'idrodinamica di Eulero, con la condizione che gli spostamenti verticali risultino trascurabili rispetto alla profondità del lago, perviene alla seguente equizione del movimento (che porta il suo nome):

$$
\frac{\delta^{2} u}{\delta t^{2}}=\underline{g} S(x) b(x) \frac{\delta^{2} u}{\delta v^{2}}
$$

dove $u=S(x) \xi$ è il volume del liquido che attraversa $S(x)$ a causa dello spostamento orizzontale, e $v=\int b(x) d x$ è l'area della super: ficie del lago compresa tra l'origine e la sezione considerata. Inoltre egli considera la funzione $\sigma(v)=S(x) b(x)$ e riportati i valori di $v(x)$ sulla ascissa e i corrispondenti $\sigma(v)$ sull'ordinata, traccia la "curva normale » o curva di Chrystal che rappresenta la sezione del lago ridotto.

Poiché una sessa è una oscillazione stazionaria, la “ $u$ ) è una funzione periodica e si può mettere nella forma

$$
\boldsymbol{u}=\boldsymbol{P} \operatorname{sen} n(t-\tau)
$$

dove $P$ è una funzione di $v$. 

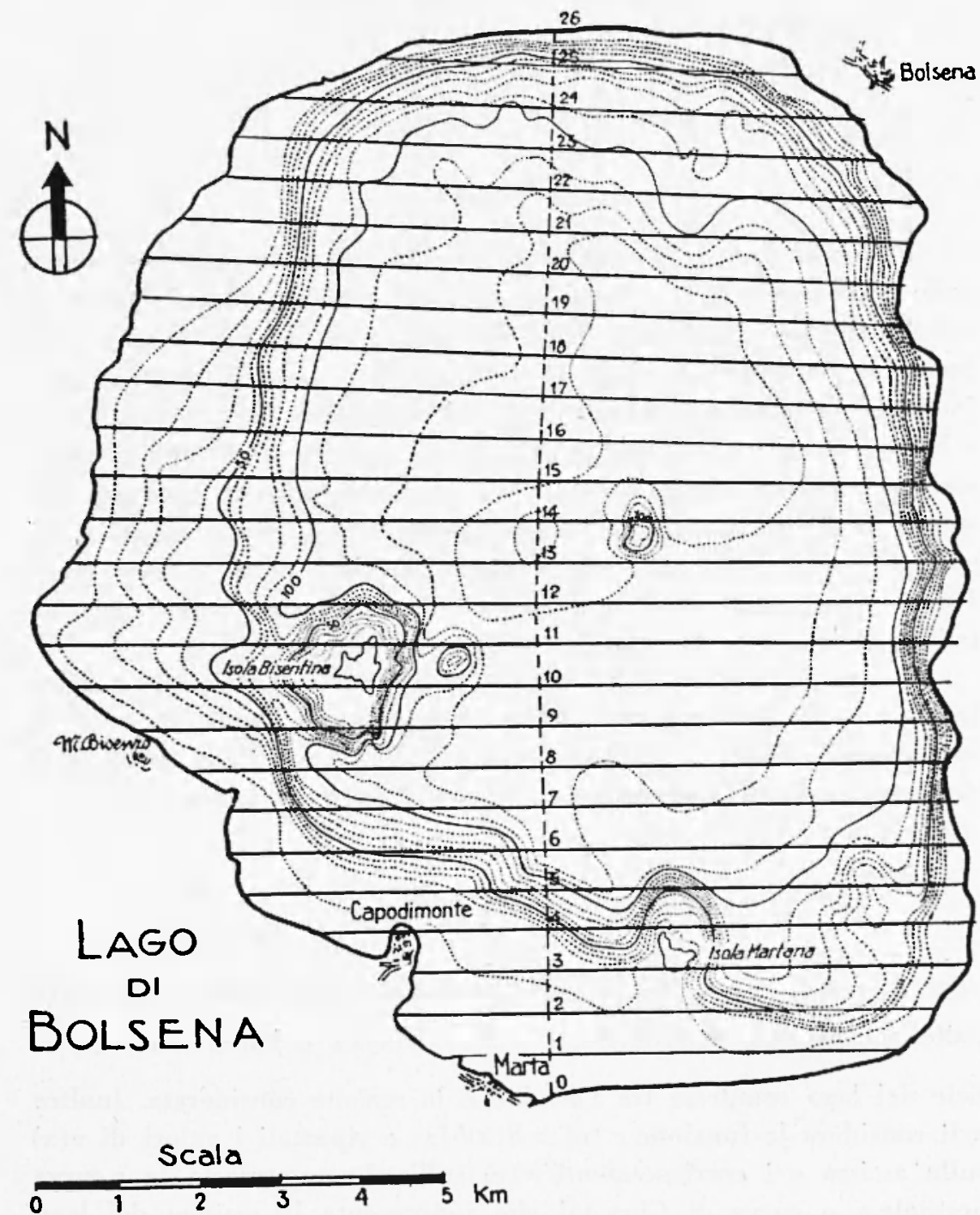

Fig. 1 
Nel caso che si tratti di un lago di ampiezza " $b$ " costante, di sezione rettangolare ma di profondità variabile, tale che $S(x)=b h(x)$, supponendo che si abljia a che fare con oscillazioni stazionarie, la [1] diviene

$$
\frac{\delta^{2} P}{\delta x^{2}}+\frac{n^{2}}{g h(x)} P=0
$$

Se poi si considera il caso particolare di un lago parabolico completo, simmetrico e concavo, e l'origine degli assi si sposta al centro del lago e si indica con $h$ la massima profondità e con a la semilunghezza del lago ridotto (fig. 2 ), allora

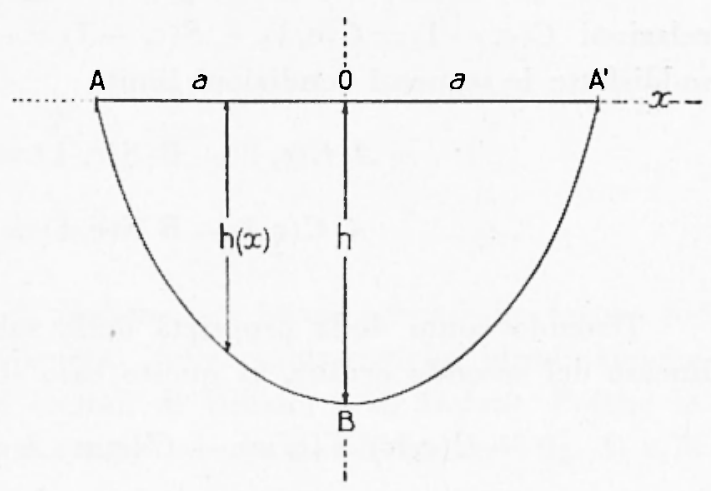

Fig. 2

$$
h(x)=h \cdot\left(1-\frac{x^{2}}{a^{2}}\right)
$$

Ponendo $\|=\frac{x}{a}$ e $c=\frac{n^{2} a^{2}}{g h}$ la $[2]$ assume la forma

$$
\left(1-u^{2}\right) \frac{\delta^{2} P}{\delta u^{2}}+c P=0
$$

e questa, introducendo le funzioni delle stesse, ci dà per " $u$ " l'espressione

$$
\vdots h\left(1-w^{*}\right)=\|=\{A C(r, w)+B S(c, w)\} \operatorname{sen} n(t-\tau)
$$

nclla quale $A$ \& $B$ sono costanti arbitrarie e $C(c, w)$ e $S(c, w)$ sono date dalle

$$
\begin{aligned}
& C(c, w)=1-\frac{c}{\cdot 2} u^{2}+\frac{c(c-1.2)}{1.2 \times 3.4} u^{4}-\ldots \\
& S(c, w)=w-\frac{c}{2 \cdot 3} u^{3}+\frac{c(c-2.3)}{2.3 \times 4.5} u^{5}-\ldots
\end{aligned}
$$


Gli spostamenti verticali risultano di conseguenza

$$
\zeta=-\frac{\delta u}{\delta x}=-\frac{1}{a} \frac{\delta u}{\delta w}=-\frac{1}{a} ; A C^{\prime}(c, w)+B S^{\prime}(c, w)\{\operatorname{sen} n(t-\tau)
$$

dove $C^{\prime}(c, w)$ e $S^{\prime}(c, w)$ sono le derivate delle funzioni delle sesse rispetto a $w$.

Dalla [4] risulta che $u=0$ per $w=+1$ e dato che sussistono le relazioni $C(c,-1)=C(c, 1)$ e $S(c,-1)=-S(c, 1)$, debbono essere soddisfatte le seguenti condizioni limite

$$
\begin{aligned}
& A C(c, 1)+B S(c, 1)=0 \\
& A C(c, 1)-B S(c, 1)=0
\end{aligned}
$$

Tenendo conto della proprietà delle soluzioni di una equazione lineare del secondo ordine, in questo caso si può scrivere

$$
C(c, w) S^{\prime}(c, w)-C^{\prime}(c, w) S(c, w)=1 \text {; }
$$

risulta quindi che $C(c, 1)$ e $S(c, 1)$ non possono essere nulle contemporaneamente e pertanto o

$$
B=0, C(c, 1)=0,
$$

oppure

$$
A=0, S(c, 1)=0 \text {, }
$$

Quando $c$ assume uno dei valori non frazionari $1 \cdot 2 ; 2 \cdot 3 ; 3 \cdot 4 ; \ldots$ $v \cdot(v+1)$, alternativamente le $C(c, w)$ e $S(c, w)$ si annullano o si riducono a funzioni intere razionali di $w$.

In queste condizioni è pertanto possibile calcolare i valori delle ampiezze degli spostamenti verticali $\zeta$ che saranno dati dalle relazioni

$$
\begin{gathered}
\zeta=-\frac{A}{a} C^{\prime}\left(c_{2 \mathrm{~S}-1} ; w\right) \text { sen } n_{2 \mathrm{~s}-1}(t-\tau) \\
\bar{\zeta}=-\frac{B}{a} S^{\prime}\left(c_{2 \mathrm{~S}} ; w\right) \text { sen } n_{\mathrm{2} \mathrm{S}}(t-\tau)
\end{gathered}
$$

rispettivamente per le ampiezze delle sesse dispari e pari. 
I periodi relativi si possono ottenere facilmente dalla

$$
\boldsymbol{T}_{v}=\frac{2 \pi}{n_{v}}
$$

e ricordando che

$$
c_{v}=\frac{n_{v}^{2} a^{2}}{g h}
$$

si ha

$$
T_{*}=\frac{2 \pi a}{\sqrt{c_{v} g}}
$$

con $c_{v}=v(v+1)$ dove $v=1,2,3 \ldots$

Applicazione al lago di Bolsena. - In un precedente lavoro sono stati determinati gli elementi delle caratteristiche idrodinamiche del lago di Bolsena con i metodi di Hidaka e di Defant. Poiché la curva di Chrystal è molto prossima ad una parabola (vedi fig. 3) e la

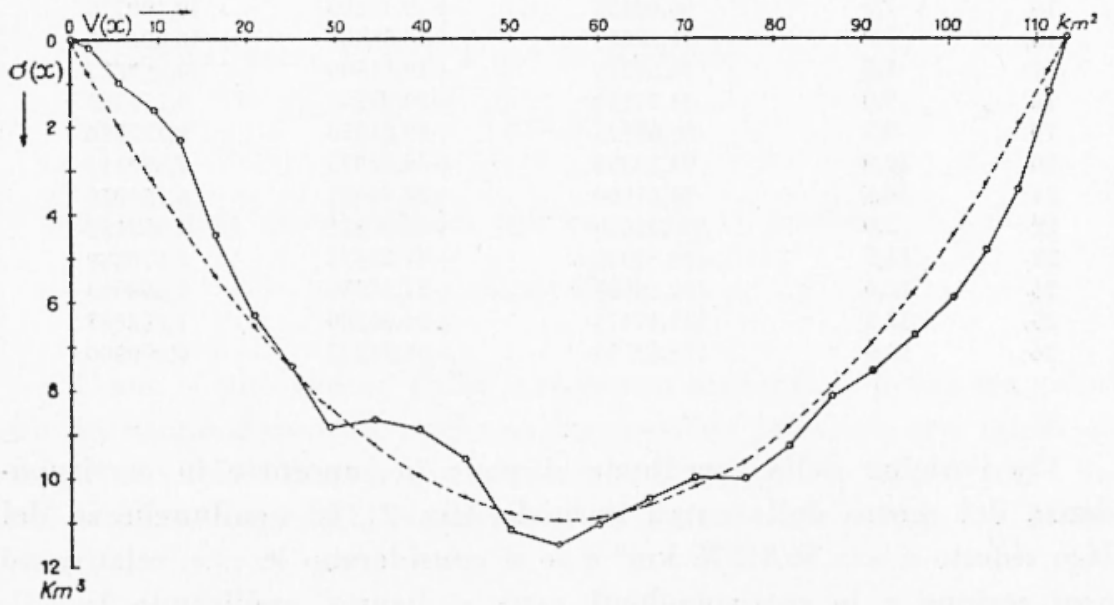

Fig. 3

forma del lago assimilabile ad un rettangolo, è stato possibile applicare per la prima volta il metodo proposto da Chrystal, nel caso di un lago parabolico completo.

Nella tabella I sono riportati i dati adoperati nel primo lavoro e che sono stati rilevati dalla carta del lago di Bolsena dell'Atlante dei 
laghi italiani di De Agostini, ove sono state tracciate 25 sezioni, distanti l'una dall'altra $500 \mathrm{~m}$ e normali alla linea alla superficie, assunta come asse delle $x$.

\section{TABELLA I}

\begin{tabular}{|c|c|c|c|c|}
\hline $\begin{array}{c}\text { sez. } \\
\text { N. }\end{array}$ & $\begin{array}{c}\mathrm{d} \\
\mathrm{km}\end{array}$ & $\begin{array}{l}\prime(x) \\
\mathrm{km}^{2}\end{array}$ & $\stackrel{x}{K_{11}}$ & $\begin{array}{l}\sigma(v) \\
\mathrm{km}^{3}\end{array}$ \\
\hline $\begin{array}{r}0 \\
1 \\
2 \\
3 \\
4 \\
5 \\
6 \\
7 \\
8 \\
9 \\
10 \\
11 \\
12 \\
13 \\
14 \\
15 \\
16 \\
17 \\
18 \\
19 \\
20 \\
21 \\
22 \\
23 \\
24 \\
25 \\
26\end{array}$ & $\begin{array}{r}0,0 \\
0,5 \\
1,0 \\
1,5 \\
2,0 \\
2,5 \\
3,0 \\
3,5 \\
4,0 \\
4,5 \\
5,0 \\
5,5 \\
6,0 \\
6,5 \\
7,0 \\
7,5 \\
8,0 \\
8,5 \\
9,0 \\
9,5 \\
10,0 \\
10,5 \\
11,0 \\
11,5 \\
12,0 \\
12,5 \\
13,0\end{array}$ & $\begin{array}{r}0,00000 \\
2,21950 \\
5,47150 \\
8,87825 \\
12,38825 \\
16,37375 \\
20,75475 \\
25,19375 \\
29,36450 \\
34,37125 \\
39,53300 \\
44,74500 \\
50,19700 \\
55,64250 \\
60,01850 \\
66,00 \cdot 75 \\
71,38075 \\
76,62775 \\
81,77150 \\
86,63125 \\
91,33350 \\
95,77900 \\
100,41600 \\
104,33900 \\
108,08525 \\
111,47475 \\
113,62550\end{array}$ & $\begin{array}{r}-56,81275 \\
-54,59325 \\
--51,34125 \\
-47,93450 \\
-44,42450 \\
-40,43900 \\
-36,05800 \\
-31,61900 \\
-27,44825 \\
--22,44150 \\
-17,27975 \\
-12,06775 \\
-6,61575 \\
-1,17025 \\
+3,20575 \\
+9,19200 \\
+14,56800 \\
+19,81500 \\
+24,95875 \\
+-29,81850 \\
+34,52075 \\
+38,96625 \\
+43,60325 \\
+47,52625 \\
+51,27250 \\
+54,66200 \\
+-56,81275\end{array}$ & $\begin{array}{r}0,000000 \\
0,203290 \\
0,992445 \\
1,56+1414 \\
2,303440 \\
4,526775 \\
6,301200 \\
7,493048 \\
8,862042 \\
8,701275 \\
8,900899 \\
9,592736 \\
11,226815 \\
11,53+1864 \\
10,961528 \\
10,490775 \\
10,094545 \\
10,329237 \\
9,271762 \\
8,122936 \\
7,598+14 \\
6,754010 \\
5,958452 \\
4.870289 \\
3,468000 \\
1,258687 \\
0,000000\end{array}$ \\
\hline
\end{tabular}

Se l'origine delle coordinate si pone in superficie in corrispondenza del centro della curva normale (fig. 2), la semilunghezza del lago ridotto è $a=56,81275 \mathrm{~km}^{2}$ e se si considerano le $v(x)$ relative ad ogni sezione e le corrispondenti $\sigma(v)$, si hanno, applicando la [3], 27 relazioni dalle quali, con il metodo dei minimi quadrati, si perviene alla relazione

$$
h(x)=10,972-0,0034 x^{2}
$$

da cui risulta per la massima profondità del lago ridotto il valore

$$
h=10,972
$$


Nella fig. 3 la parabola trovata è posta in confronto con la " curva normale " ed è messa in evidenza la buona approssimazione prevista.

Periodi. Calcolato il valore di $h$ e noto $a$, prendendo per l'accelerazione di gravità il valore $g=0,009803 \mathrm{~km} / \mathrm{sec}^{2}$, tenendo conto del valore di $c$ per la sessa che si considera, dalla relazione

$$
T=2 \pi \frac{a}{\sqrt{c_{v} h},}
$$

con $c_{v}=v(v+1)$ e $v=1,2,3 \ldots$ è facile determinare $\mathrm{i}$ vari periodi.

Infatti, nell'espressione del periodo, posto i valori detti di $a, h, g$ e con $c_{1}=1 \cdot 2$, si ottiene per il periodo della uninodale il valore

$$
T_{1}=12^{\mathrm{m}}, 83
$$

mentre per $c=2 \cdot 3$ il periodo della binodale risulta

$$
T_{2}=7^{\mathrm{m}}, 41
$$

Ponendo invece $c_{3}=3 \cdot 4$ per la trinodale

$$
T_{3}=5^{\mathrm{m}}, 24
$$

e infine per la quadrinodale, con $c_{4}=4 \cdot 5$, si perviene al valore

$$
T_{4}=4^{m}, 06
$$

Comı si può vedere dallo specchietto seguente, $\mathrm{i}$ primi tre valori

\begin{tabular}{|c|c|c|c|c|}
\hline \multicolumn{4}{|c|}{ Period $i$} & \multirow{5}{*}{$\begin{array}{c}\text { metodo d } \\
"\end{array}$} \\
\hline unidodale & binodale & trinodale & quadrinodale & \\
\hline $12^{m, 83}$ & $7^{m}, 41$ & $5^{m}, 24$ & $4^{m}, 06$ & \\
\hline 13,05 & 7,90 & 5,40 & & \\
\hline 13,15 & 8,20 & 5.35 & & \\
\hline 13,23 & 7,92 & 5,40 & 3,80 & $\begin{array}{l}\text { medi osservati regi- } \\
\text { strazioni Palazzo. }\end{array}$ \\
\hline
\end{tabular}
trovati vanno d'accordo, anche se leggermente inferiori, con quelli ottenuti con i due metodi precedentemente applicati e altresi con i valori medi rilevati dalle registrazioni di Palazzo e riportati nel lavoro citato. 
L'ultimo valore ottenuto è molto prossimo al periodo $3^{\text {n1 }}, 8$ rilevato come media di due valori dalla registrazione di Palazzo a Bolsena, valore ehe nell'altra nota si dava appunto come probabile periodo della quadrinodale.

Spostamenti verticali e nodi. Gli spostamenti verticali lungo la sezione del lago sono stati ottenuti applicando le formule [6] e ponendo in esse $i$ valori di $c$ relativi ad ogni sessa.

Per l'uninodale infatti

$$
\zeta=-\frac{A}{a} C^{\prime}\left(c_{1}, w\right) \operatorname{sen} n_{1}(t-\tau)
$$

dove per $c_{1}=1 \cdot 2$

$$
C\left(c_{1}, w\right)=1-w^{2}
$$

e ricordando che $w=\frac{x}{a}$, si ha in definitiva

$$
\zeta=\frac{2 A x}{a^{2}} \operatorname{sen} n_{1}(t-\tau)
$$

Posto come al solito $\zeta=1$ all'estremo del lago si sono ottenuti, per le varie sezioni, i valori delle ampiezze riportati nella tabella $\Pi$. L'uninodo cade nel centro del lago ridotto in corrispondenza di una distanza di $6,634 \mathrm{~km}$ da Marta.

Per la binodale gli spostamenti verticali sono dati dalla relazione

$$
\zeta=-\frac{B}{a} S^{\prime}\left(c_{a}, w\right) \operatorname{sen} n_{9}(t-\tau)
$$

dove per $c_{2}=2 \cdot 3$ si ha dalla seconda delle [6]

$$
S\left(c_{\mathrm{i}}, w\right)=w-u^{3}
$$

derivando e sostituendo nella espressione di $\zeta$, risulta

$$
\zeta=-\frac{B}{a}\left(\frac{a^{\bar{z}}-3 x^{\circ}}{a^{2}}\right) \operatorname{sen} n_{9}(t-\tau)
$$

I binodi si hanno se $\zeta=0$ e precisamente per $a^{2}-3 x^{2}=0$ da cui risultano $d^{\mathrm{II}}=3,367 \mathrm{~km}$ e $d^{\mathrm{II}}{ }_{2}=9,817 . \mathrm{km}$, sempre contati a partire da Marta. 


\section{TABELLA II}

\begin{tabular}{|c|c|c|c|c|}
\hline $\begin{array}{c}\text { ¿ez. } \\
\text { N. }\end{array}$ & unidodale & binodale & Irinodale & quadrinodale \\
\hline 0 & +1 & +1 & +1 & +1 \\
\hline 1 & 0,961 & $0,88 \pi$ & 0,777 & 0,645 \\
\hline 2 & $0,90.1$ & 0,725 & 0,489 & $+0,230$ \\
\hline 3 & $0,81.4$ & 0,568 & 0,236 & $-0,077$ \\
\hline 4 & 0,782 & 0,417 & $+0,022$ & 0,282 \\
\hline 5 & 0,712 & 0,260 & $-0,166$ & 0,402 \\
\hline 6 & 0,635 & $+0,104$ & 0,313 & 0,426 \\
\hline 7 & 0,557 & —0,035 & 0,404 & 0,367 \\
\hline 8 & 0,183 & 0,150 & 0,443 & 0,262 \\
\hline 9 & 0,395 & 0,266 & 0,438 & $-0,104$ \\
\hline 10 & $0,30.1$ & 0,361 & 0,386 & $+0,066$ \\
\hline 11 & 0,212 & 0,432 & 0,295 & 0,215 \\
\hline 12 & 0,116 & 0,480 & 0,171 & 0,325 \\
\hline 13 & $+0,021$ & 0,499 & $-0,031$ & 0,373 \\
\hline 14 & $--0,056$ & 0,495 & $+0,084$ & 0,363 \\
\hline 15 & 0,162 & 0,461 & 0,232 & 0,280 \\
\hline 16 & 0,256 & 0,401 & 0,342 . & $+0,147$ \\
\hline 17 & 0,319 & 0,318 & 0,417 & $-0,016$ \\
\hline 18 & 0,439 & 0,211 & 0,447 & 0,186 \\
\hline 19 & 0,525 & $-0,087$ & 0,426 & 0,326 \\
\hline 20 & 0,608 & $+0,054$ & 0,349 & 0,413 \\
\hline 21 & 0,686 & 0,206 & 0,222 & 0,421 \\
\hline 22 & 0.767 & 0.384 & $+0,021$ & 0,316 \\
\hline 23 & 0,837 & 0,550 & $-0,209$ & $-0,106$ \\
\hline $2 !$ & 0,902 & 0,722 & 0,484 & $+0,223$ \\
\hline 25 & 0,962 & 0,889 & 0,783 & 0,653 \\
\hline 26 & 1,000 & 1,000 & 1,000 & 1.000 \\
\hline
\end{tabular}

Se si sostituisce $c_{3}=3 \cdot 4$ nella prima delle [6], si ha

$$
C\left(c_{3}, w\right)=1-6 w^{2}+5 u^{3}
$$

e quindi, per la trinodale, l'andamento degli spostamenti verticali risulta dalla

$$
\zeta=-\frac{A}{a^{4}}\left(-12 a^{\prime \prime} x+20 x^{3}\right) \operatorname{sen} n_{3}(t-\tau)
$$

I tre punti nodali si avranno al solito risolvendo la

$$
20 x^{3}-12 a^{2} x=0 .
$$

I due trinodi estremi risultano così distanti da Marta rispettivamentre $d_{1}{ }^{\mathrm{II}}=2,052 \mathrm{~km}$ e $d_{: !}{ }^{\mathrm{III}}=11,051 \mathrm{~km}$, mentre quello centrale coincide con quello della uninodale. 
Per la quadrinodale, dalla seconda delle [6] si ottiene l'andamento delle ampiezze verticali, considerando $c_{4}=4 \cdot 5$ : allora

$$
\begin{aligned}
& S\left(c_{1}, w\right)=\frac{w}{\mathfrak{\jmath}}\left(1-u^{2}\right)\left(3-7 u^{2}\right), \\
& S^{\prime}\left(c_{1}, w\right)=\frac{1}{3}\left(3-30 u^{2}-35 u^{1}\right),
\end{aligned}
$$

perciò

$$
\dddot{\imath}=-\frac{B}{3 a^{5}}\left(3 a^{4}-39 a^{2} x^{2}+35 x^{4}\right) \operatorname{sen} n_{4}(t-\tau) .
$$

I nodi si hanno risolvendo la biquadratica fra parentesi e risultano cosi ubicati :

$$
\begin{array}{ll}
d_{1}{ }^{\mathrm{IV}}=1,355 \mathrm{Km} & d_{\mathrm{a}}{ }^{\mathrm{V}}=4,863 \mathrm{Km} \\
d_{\mathrm{i}}{ }^{\mathrm{IV}}=8,452 \mathrm{Km} & d_{t}{ }^{\mathrm{Iv}}=11,686 \mathrm{Km},
\end{array}
$$

dall'estremo sud del lago.

Nella fig. 4 sono rappresentati gli andamenti delle prime quattro sesse del lago di Bolsena e risulta evidente che verso l'estremità del

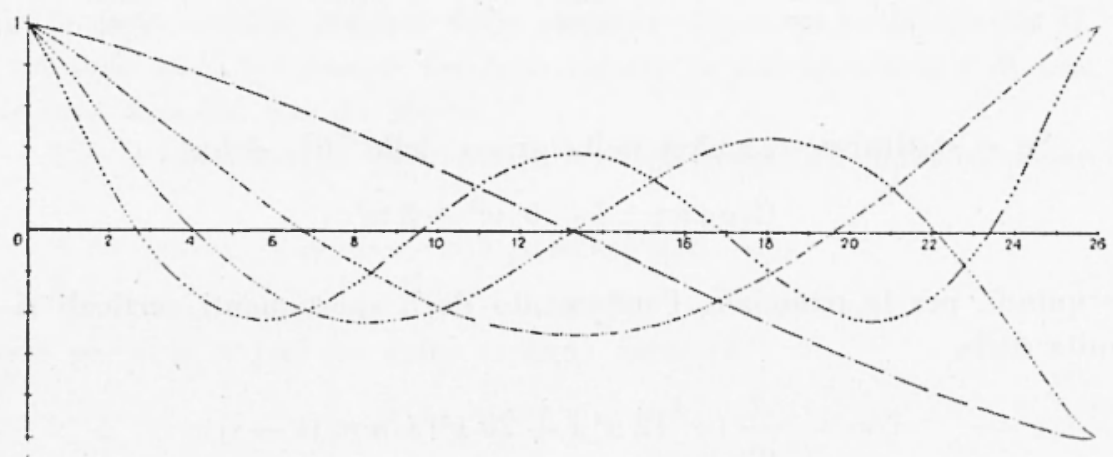

Fig. 4

lago si accorcia la loro lunghezza d'onda. Inoltre può essere notato che le ampiezze verticali delle sesse sono maggiori all'estremità che non al centro del lago o in vicinanza di esso. A parità di ampiezza agli estremi, nella parte centrale del lago ha ampiezza minore la sessa di lunghezza d'onda maggiore. 
La particolarità della forma di questo lago spiega in parte la frequenza e l'ampiezza rilevante delle sesse, tanto da essere osservate anche ad occhio; sicché, diventa sempre più interessante eseguire una nuova serie di registrazioni, con vari limnografi opportunamente distribuiti intorno al lago.

Roma - Istituto Nazionale di Geofisica - Maggio 1953

\section{RIASSUNTO}

Si applica per la prima volta, dato l'andamento della curva normale del lago di Bolsena, il metodo proposto da Chrystal per la determinazione degli elementi delle caratteristiche idrodinamiche di un lago parabolico completo. Si determinano gli andamenti degli spostamenti verticali, $i$ nodi $e$ i periodi delle prime quattro sesse.

$I$ risultati concordano con quelli ottenuti, in un precedente lavoro, con $i$ metodi di Hidaka e di Defant e con i dati rilevati dalle registrazioni ottenute da Palazzo.

\section{SUMMARY}

For the first time, given the behavior of the normal curve of the Lake of Bolsena, the method proposed by Crystal for the determination of the characteristic hydrodynamic elements of a complete parabolic lake has been applied. The behavior of the vertical displacements, the nodes and the periods of the first four seiches have been determined with this method.

The results agree with those obtained in a preceding work employing the methods of Hidaka and Defant, and with the data evaluated from the registrations obtained by Palazzo.

\section{BIBLIOGRAFIA}

Chnystal G.: On the hydrodinamical Theory of Seiches. Transactions of the Royal Society of Edinburgh XLI (1905), XLV (1906), XLVI (1907-8).

Palazzo L.: Studi limnologici sul lago di Bolsena. Atti del V Congresso Geografico Italiano. Vol. II, Sez. 1 (1904).

Cator P.: Le Sesse del lugo di Garda. Annali di Geofisica. Vol. I (1948).

Di Filirpo D.: Le Sesse del lago di Bolsena. Annali di Geofisica. Vol. IV (1951). 